\title{
Weed Management in Clover -- 20051
}

\author{
J. A. Ferrell ${ }^{2}$
}

Table 1. Weed Management in Clover.



1. This document is SS-AGR-235, one of a series of the Agronomy Department, Florida Cooperative Extension Service, Institute of Food and Agricultural Sciences, University of Florida. Published January 2005. Please visit the EDIS Website at http://edis.ifas.ufl.edu.

2. J. A. Ferrell, assistant professor, Agronomy Department and Florida Cooperative Extension Service, Institute of Food and Agricultural Sciences, University of Florida, Gainesville, 32611.

The use of trade names in this publication is solely for the purpose of providing specific information. UF/IFAS does not guarantee or warranty the products named, and references to them in this publication does not signify our approval to the exclusion of other products of suitable composition. Use herbicides safely. Read and follow directions on the manufacturer's label.

The Institute of Food and Agricultural Sciences is an equal opportunity/affirmative action employer authorized to provide research, educational information and other services only to individuals and institutions that function without regard to race, color, sex, age, handicap, or national origin. For information on obtaining other extension publications, contact your county Cooperative Extension Service office. Florida Cooperative Extension Service/Institute of Food and Agricultural Sciences/University of Florida/Christine Taylor Waddill, Dean. 
Table 1. Weed Management in Clover.

\begin{tabular}{|c|c|c|}
\hline $\begin{array}{l}\text { Trade Name and } \\
\text { Rate of } \\
\text { Commercial } \\
\text { Product Per Acre }\end{array}$ & $\begin{array}{l}\text { Common Name and } \\
\text { Rate in Pounds of } \\
\text { Active Ingredient } \\
\text { Per Acre }\end{array}$ & Remarks \\
\hline $\begin{array}{l}\text { Butyrac } 200 \\
2.3 \text { qts } \\
\text { or } \\
\text { Butoxone } \\
4.3 \text { to } 6.5 \text { pt }\end{array}$ & $2,4-\mathrm{DB}$ & $\begin{array}{l}\text { For postemergence control of winter or summer annual broadleaf weeds, } \\
\text { spray emerged weeds less than } 3 \text { inches tall. } 2,4 \text {-DB will not control wild } \\
\text { radish, chickweed, or mustards. Do not graze or cut for hay for } 60 \text { days } \\
\text { after application. Do not apply to clovers grown for seed. Rainfall or } \\
\text { irrigation within } 7 \text { to } 10 \text { days after treatment can cause injury. DO NOT add } \\
\text { wetting agents or surfactants to the spray mix. Apply after clover is in the } 2 \\
\text { to } 4 \text { trifoliate leaf stage. }\end{array}$ \\
\hline \multicolumn{3}{|c|}{ Postemergence - Established Clover } \\
\hline $\begin{array}{l}\text { Kerb } 50 \mathrm{~W} \\
1 \text { to } 1.5 \mathrm{lb}\end{array}$ & pronamide & $\begin{array}{l}\text { Controls winter annual grasses and some broadleaf weeds in clovers, } \\
\text { birdsfoot trefoil and crown vetch. Apply from November through February. } \\
\text { DO NOT graze or harvest for hay for } 120 \text { days after application. KERB is a } \\
\text { restricted use herbicide. }\end{array}$ \\
\hline
\end{tabular}

Table 2. Estimated effectiveness of recommended herbicides on common weeds in Florida Clover.

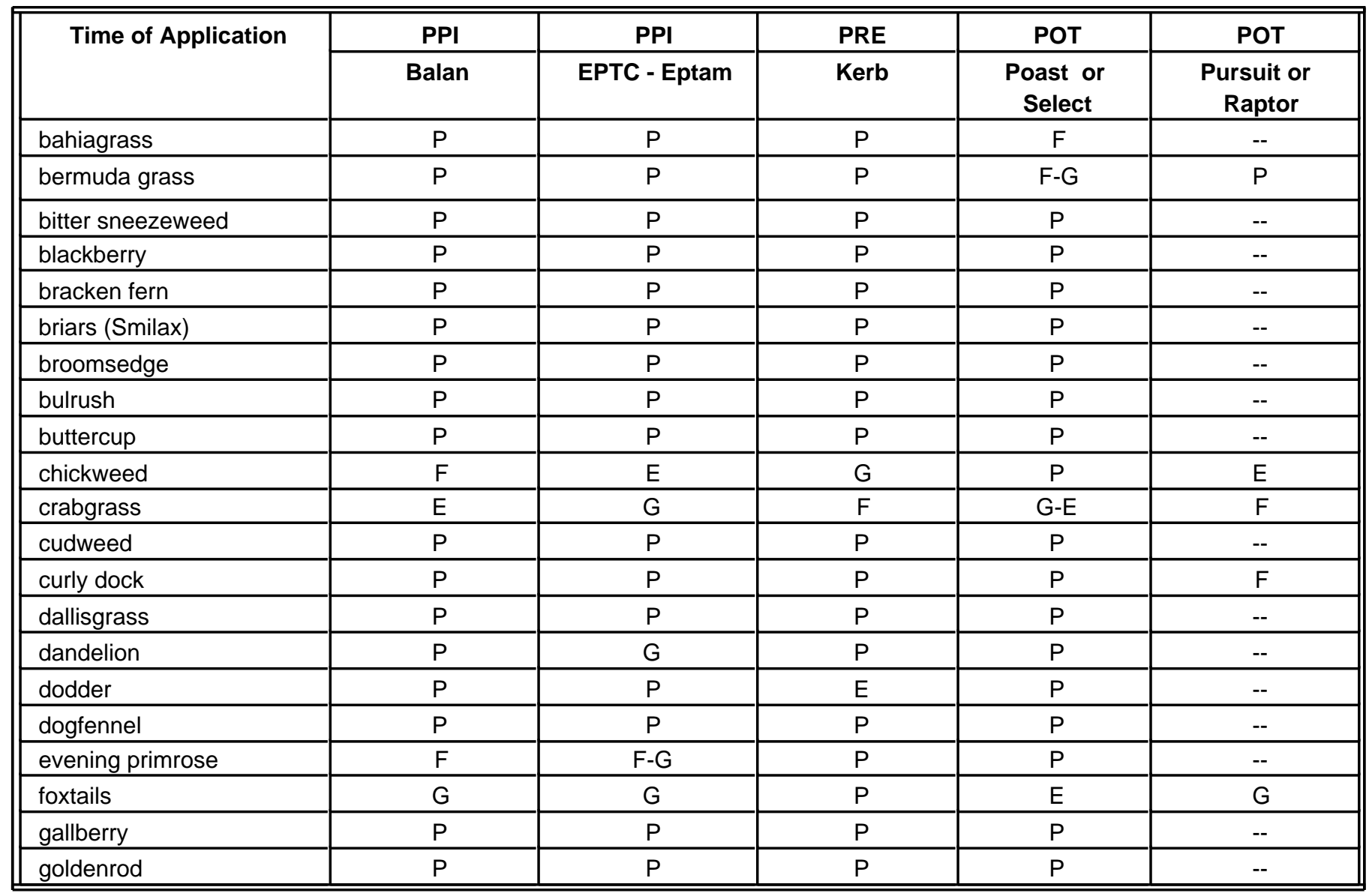


Table 2. Estimated effectiveness of recommended herbicides on common weeds in Florida Clover.

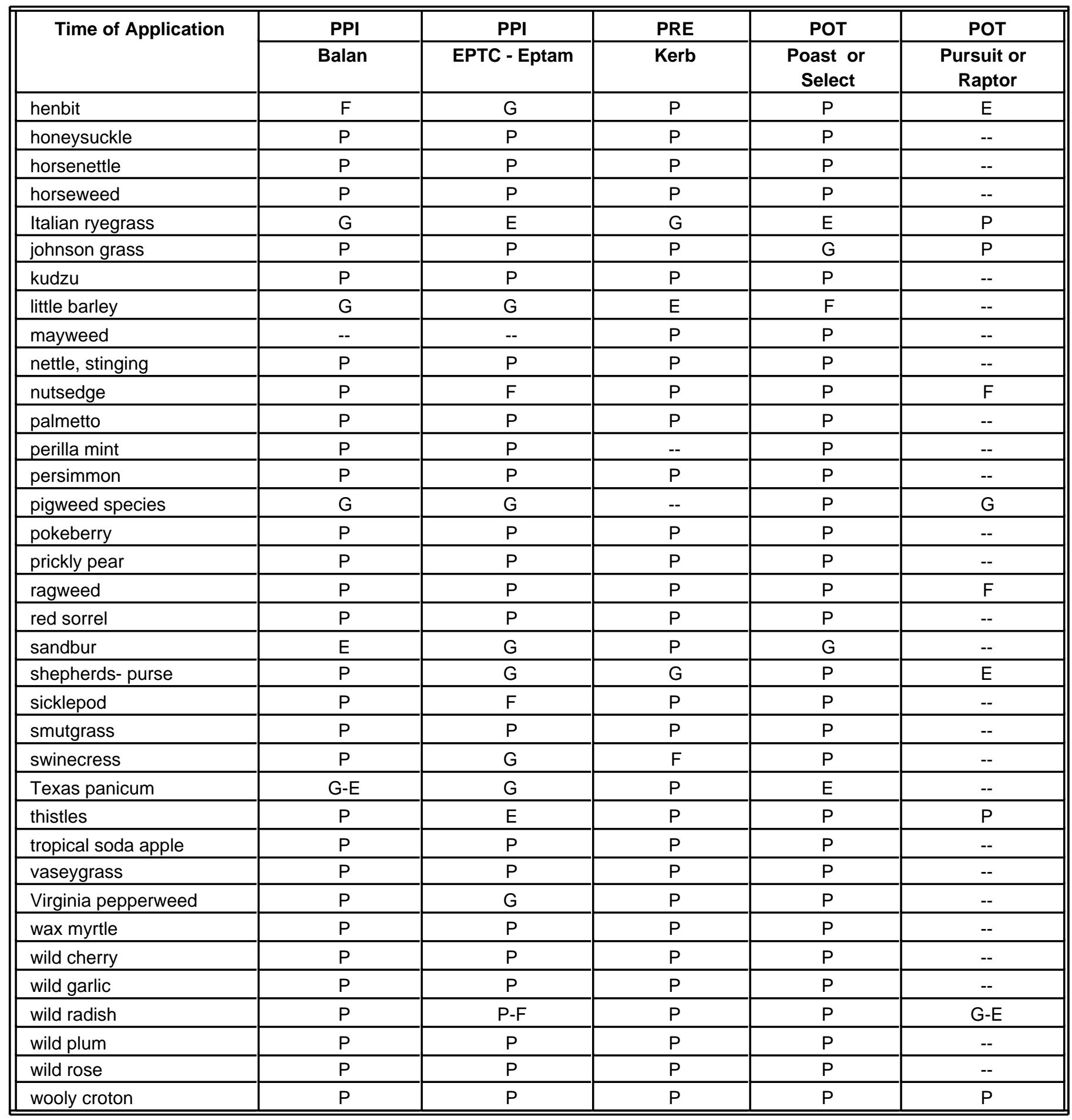

Table 3. Estimated effectiveness of recommended herbicides on common weeds in Florida Clover (continued).

\begin{tabular}{|l|c|c|c|c|c||}
\hline \hline $\begin{array}{l}\text { Time of } \\
\text { Application }\end{array}$ & POT & POT & POT & POT & POT \\
\hline & Velpar & $\begin{array}{c}\text { Sencor or } \\
\text { Lexone }\end{array}$ & $\begin{array}{c}\text { Paraquat- } \\
\text { Gramoxone Max }\end{array}$ & Buctril & 2,4-D \\
\hline bahiagrass & $\mathrm{P}$ & $\mathrm{P}$ & $\mathrm{P}$ & $\mathrm{P}$ & $\mathrm{P}$ \\
\hline bermudagrass & $\mathrm{P}$ & $\mathrm{P}$ & $\mathrm{P}$ & $\mathrm{P}$ & $\mathrm{P}$ \\
\hline bitter sneezeweed & -- & -- & -- & $\mathrm{E}$ & $\mathrm{G}$ \\
\hline \hline
\end{tabular}


Table 3. Estimated effectiveness of recommended herbicides on common weeds in Florida Clover (continued).

\begin{tabular}{|c|c|c|c|c|c|c|}
\hline $\begin{array}{l}\text { Time of } \\
\text { Application }\end{array}$ & РOT & РOT & РOT & РОТ & РOT & РOT \\
\hline & Velpar & $\begin{array}{c}\text { Sencor or } \\
\text { Lexone }\end{array}$ & $\begin{array}{c}\text { Paraquat- } \\
\text { Gramoxone Max }\end{array}$ & Buctril & 2,4-D & 2,4-DB \\
\hline blackberry & $\mathrm{F}$ & $\mathrm{P}$ & $\mathrm{P}$ & $\mathrm{P}$ & $\mathrm{F}$ & $\mathrm{P}$ \\
\hline bracken fern & $\mathrm{F}$ & -- & $\mathrm{P}$ & -- & $\mathrm{P}$ & $\mathrm{P}$ \\
\hline briars (Smilax) & $\mathrm{F}$ & $\mathrm{P}$ & $\mathrm{P}$ & $\mathrm{P}$ & $\mathrm{P}$ & $P$ \\
\hline broomsedge & $\mathrm{P}$ & $\mathrm{P}$ & $\mathrm{P}$ & $\mathrm{P}$ & $\mathrm{P}$ & $\mathrm{P}$ \\
\hline bulrush & -- & -- & $\mathrm{P}$ & $\mathrm{P}$ & $\mathrm{G}$ & $\mathrm{P}$ \\
\hline buttercup & $\mathrm{G}$ & $\mathrm{G}$ & $\mathrm{G}$ & -- & $E$ & $\mathrm{~F}$ \\
\hline chickweed & $E$ & $E$ & $E$ & $\mathrm{~F}$ & $\mathrm{~F}$ & $\mathrm{P}$ \\
\hline crabgrass & $\mathrm{P}$ & $\mathrm{F}$ & $\mathrm{F}$ & $\mathrm{P}$ & $\mathrm{P}-\mathrm{F}$ & $\mathrm{P}$ \\
\hline cudweed & -- & -- & $\mathrm{G}$ & $\mathrm{P}$ & $\mathrm{F}$ & -- \\
\hline curly dock & $\mathrm{P}$ & -- & $\mathrm{P}$ & -- & $\mathrm{F}$ & $\mathrm{P}$ \\
\hline dallisgrass & -- & $\mathrm{P}$ & $\mathrm{P}$ & $\mathrm{P}$ & $\mathrm{P}$ & $\mathrm{P}$ \\
\hline dandelion & $E$ & $\mathrm{G}$ & $\mathrm{G}$ & $\mathrm{P}$ & $E$ & $\mathrm{G}$ \\
\hline dodder & -- & -- & $\mathrm{G}-\mathrm{E}$ & -- & $\mathrm{P}$ & $\mathrm{P}$ \\
\hline dogfennel & -- & -- & $\mathrm{P}$ & $\mathrm{P}$ & $\mathrm{F}$ & $\mathrm{P}$ \\
\hline $\begin{array}{l}\text { evening } \\
\text { primrose }\end{array}$ & $E$ & G & P-F & -- & $E$ & $\mathrm{G}$ \\
\hline foxtails & -- & $\mathrm{P}$ & $\mathrm{F}$ & $\mathrm{P}$ & $\mathrm{P}$ & $P$ \\
\hline gallberry & $\mathrm{P}$ & $\mathrm{P}$ & $\mathrm{P}$ & $\mathrm{P}$ & $\mathrm{G}$ & $\mathrm{P}$ \\
\hline goldenrod & -- & -- & $\mathrm{P}$ & $\mathrm{P}$ & $\mathrm{F}$ & $\mathrm{P}$ \\
\hline henbit & $E$ & $E$ & $\mathrm{G}$ & $\mathrm{F}$ & $\mathrm{F}$ & $\mathrm{P}$ \\
\hline honeysuckle & -- & $\mathrm{P}$ & $\mathrm{P}$ & $\mathrm{P}$ & $E$ & $\mathrm{P}$ \\
\hline horsenettle & -- & $\mathrm{P}$ & $\mathrm{P}$ & $\mathrm{P}$ & $\mathrm{P}$ & $\mathrm{P}$ \\
\hline horseweed & -- & $\mathrm{P}$ & $\mathrm{P}$ & $\mathrm{P}$ & $\mathrm{F}$ & $\mathrm{P}$ \\
\hline Italian ryegrass & $\mathrm{G}$ & $\mathrm{P}$ & G-E & $\mathrm{P}$ & $\mathrm{P}$ & $\mathrm{P}$ \\
\hline johnsongrass & -- & $P$ & $P$ & $P$ & $P$ & $P$ \\
\hline kudzu & -- & $\mathrm{P}$ & $\mathrm{P}$ & $\mathrm{P}$ & $\mathrm{P}-\mathrm{F}$ & $\mathrm{P}$ \\
\hline little barley & $E$ & $\mathrm{P}$ & G-E & $\mathrm{P}$ & $\mathrm{P}$ & $\mathrm{P}$ \\
\hline mayweed & $F-G$ & -- & $\mathrm{E}$ & -- & $\mathrm{F}$ & $\mathrm{P}$ \\
\hline nettle, stinging & -- & -- & -- & -- & $\mathrm{P}$ & $\mathrm{P}$ \\
\hline nutsedge & $\mathrm{P}$ & $\mathrm{P}$ & $\mathrm{P}$ & $\mathrm{P}$ & $\mathrm{P}$ & $\mathrm{P}$ \\
\hline palmetto & $\mathrm{P}$ & $\mathrm{P}$ & $\mathrm{P}$ & $\mathrm{P}$ & $\mathrm{P}$ & $\mathrm{P}$ \\
\hline perilla mint & -- & -- & -- & -- & $\mathrm{P}$ & -- \\
\hline persimmon & $\mathrm{F}$ & $\mathrm{P}$ & $\mathrm{P}$ & $\mathrm{P}$ & $\mathrm{P}$ & $\mathrm{P}$ \\
\hline pigweed & $\mathrm{G}$ & $\mathrm{G}$ & $\mathrm{G}$ & $\mathrm{F}$ & $\mathrm{F}$ & $\mathrm{G}$ \\
\hline pokeberry & -- & -- & -- & $\mathrm{P}$ & $E$ & $\mathrm{G}$ \\
\hline prickly pear & $\mathrm{P}$ & $\mathrm{P}$ & $\mathrm{P}$ & $\mathrm{P}$ & $\mathrm{P}$ & $\mathrm{P}$ \\
\hline ragweed & $\mathrm{F}$ & $\mathrm{G}$ & $\mathrm{G}$ & $\mathrm{F}$ & $\mathrm{E}$ & $\mathrm{G}$ \\
\hline red sorrel & -- & -- & $\mathrm{P}-\mathrm{F}$ & -- & $\mathrm{P}$ & $\mathrm{P}$ \\
\hline sandbur & -- & $\mathrm{F}$ & $\mathrm{G}$ & $\mathrm{P}$ & $\mathrm{P}$ & $\mathrm{P}$ \\
\hline $\begin{array}{l}\text { shepherds- } \\
\text { purse }\end{array}$ & $E$ & $E$ & G & G & $E$ & $\mathrm{G}$ \\
\hline sicklepod & -- & $\mathrm{F}$ & $F-G$ & -- & $\mathrm{G}$ & $\mathrm{F}$ \\
\hline smutgrass & $\mathrm{G}$ & $\mathrm{P}$ & $\mathrm{P}$ & $\mathrm{P}$ & $\mathrm{P}$ & $\mathrm{P}$ \\
\hline
\end{tabular}


Table 3. Estimated effectiveness of recommended herbicides on common weeds in Florida Clover (continued).

\begin{tabular}{|c|c|c|c|c|c|c|}
\hline $\begin{array}{l}\text { Time of } \\
\text { Application }\end{array}$ & РОТ & РОТ & РОТ & РОТ & РОТ & РОТ \\
\hline & Velpar & $\begin{array}{c}\text { Sencor or } \\
\text { Lexone }\end{array}$ & $\begin{array}{c}\text { Paraquat- } \\
\text { Gramoxone Max }\end{array}$ & Buctril & 2,4-D & 2,4-DB \\
\hline swinecress & $E$ & $E$ & $E$ & $E$ & $E$ & $\mathrm{~F}$ \\
\hline Texas panicum & -- & $\mathrm{P}-\mathrm{F}$ & $\mathrm{G}$ & $\mathrm{P}$ & $\mathrm{P}$ & $\mathrm{P}$ \\
\hline thistles & $E$ & $\mathrm{G}$ & $\mathrm{G}$ & $\mathrm{P}$ & $E$ & $\mathrm{~F}$ \\
\hline $\begin{array}{l}\text { tropical soda } \\
\text { apple }\end{array}$ & $G$ & $\mathrm{P}$ & $P$ & $P$ & $\mathrm{P}-\mathrm{F}$ & $P$ \\
\hline vaseygrass & -- & $\mathrm{P}$ & $\mathrm{P}$ & $\mathrm{P}$ & $\mathrm{P}$ & $\mathrm{P}$ \\
\hline $\begin{array}{l}\text { Virginia } \\
\text { pepperweed }\end{array}$ & $E$ & $\mathrm{G}$ & $\mathrm{G}$ & $G$ & $\mathrm{G}$ & $E$ \\
\hline wax myrtle & $\mathrm{P}$ & $\mathrm{P}$ & $\mathrm{P}$ & $\mathrm{P}$ & $\mathrm{G}$ & $\mathrm{P}$ \\
\hline wild cherry & $E$ & $\mathrm{P}$ & $\mathrm{P}$ & $\mathrm{P}$ & $E$ & $\mathrm{P}$ \\
\hline wild garlic & -- & $\mathrm{P}$ & $E$ & $\mathrm{P}$ & G-E & $\mathrm{P}$ \\
\hline wild radish & $E$ & $E$ & $\mathrm{P}$ & $\mathrm{F}-\mathrm{G}$ & $\mathrm{G}$ & $\mathrm{P}$ \\
\hline wild plum & $E$ & $\mathrm{P}$ & $\mathrm{P}$ & $\mathrm{P}$ & $E$ & $\mathrm{P}$ \\
\hline wild rose & -- & $\mathrm{P}$ & $\mathrm{P}$ & $\mathrm{P}$ & $\mathrm{G}$ & $\mathrm{P}$ \\
\hline wooly croton & $\mathrm{P}$ & $\mathrm{P}$ & $\mathrm{P}$ & $\mathrm{P}$ & $\mathrm{G}$ & $\mathrm{P}$ \\
\hline
\end{tabular}

